\title{
Configuration space analysis of rigid mechanisms
}

Samuli Piipponen and Jukka Tuomela 


\title{
CONFIGURATION SPACE ANALYSIS OF RIGID MECHANISMS
}

\author{
SAMULI PIIPPONEN AND JUKKA TUOMELA
}

\begin{abstract}
In mechanical engineering and robotics, the main problem is usually to solve the equations of motion of a given rigid mechanism. The rigid mechanisms are sometimes restricted or controlled to move along a constrained path. In engineering sciences, the most common constraints are called ideal joint constraints. The ideal joint constraints are generally holonomic constraints involving only the coordinates and orientation of a mechanism with respect to a fixed coordinate frame. An important aspect is that ideal joint constraints can be formulated as a set of polynomial equations. This means that the configuration spaces of typical rigid mechanisms can be treated as algebraic varieties and the components of a constraint function as generators of a polynomial ideal. This geometry algebra equivalence and the advances of computational commutative algebra and algebraic geometry gives us means to actually compute the properties of configuration spaces.
\end{abstract}

2000 Mathematics Subject Classification: 58D30, 00A69,14-00,14Q99

Keywords: mechanisms, robotics, holonomic constraints, algebraic geometry, computational algebraic geometry, Gröbner basis

\section{INTRODUCTION}

The equations of motion arising from Lagrangian mechanics for multibody systems are typically differential algebraic equations (DAE) where algebraic equations in our case determine the holonomic constraints. The equations of motion in Lagrangian mechanics in the holonomic case are generally of the form

$$
\begin{gathered}
f(t, u, \dot{u}, \ddot{u}, \lambda)=0, \\
g(u)=0,
\end{gathered}
$$

where $u: I \mapsto g^{-1}(0) \subset \mathbb{R}^{k}$ is the trajectory of the system, $\lambda$ denotes the Lagrangian multipliers defining the holonomic constraint forces and $g^{-1}(0)$ is usually the analytic or algebraic variety defining the configuration space. In this paper we will introduce modern methods of computational algebraic geometry to study the configuration space as an algebraic variety. We will present relevant theorems and give three easy examples of planar mechanisms and their configuration space analysis. In computations, we have used the well established computer program SINGULAR [9]. In most of the computations the key is to compute the Gröbner bases of a given ideal in a particular monomial ordering. 


\section{PRELIMINARY DEFINITIONS}

Let $\mathbb{A}=\mathbb{K}\left[x_{1}, \ldots, x_{k}\right]$ be the ring of polynomials with coefficient field $\mathbb{K}$. We will always assume that $\mathbb{K}$ is algebraically closed. We can look at the vector components of the constraint map $g: \mathbb{R}^{k} \mapsto \mathbb{R}^{n}$ as functions or as generators of an ideal $d=$ $\left\langle g_{1}, \ldots, g_{n}\right\rangle \subset \mathbb{A}$. For standard facts about the ideal theory we refer to $[3,4,8,10]$. Since the polynomial rings that we consider are Noetherian, we know that every ideal in $\mathbb{A}$ is finitely generated. The geometric object corresponding to an ideal $\mathscr{l} \subset \mathbb{A}$ is its variety $\mathrm{V}(\mathcal{Q}) \subset \mathbb{K}^{k}$ which is the vanishing set of all polynomials in $\mathscr{C} \subset \mathbb{A}$. We will also frequently use the fact that any radical ideal can be written uniquely as a finite intersection of prime ideals. This prime decomposition implies directly the decomposition of the corresponding variety into its irreducible parts which is called the irreducible decomposition of a variety.

Let us then present the tools which allow us to do local analysis on varieties and distinguish between different types of singularities.

Definition 1. (Local ring) A local ring $R$ is a ring which has exactly one maximal ideal.

Remark 1. In this paper we look at localizations of polynomial rings with respect to a point/maximal ideal. We consider equivalence classes of polynomials giving always the same value when evaluated at $\mathrm{V}(\mathcal{l})$. The equivalence classes are given by

$$
[f]=\{g \in \mathbb{A} \mid f-g \in I(\mathrm{~V}(\mathcal{l}))\},
$$

where $I(\mathrm{~V}(\mathscr{\ell}))$ denotes the ideal of a variety $\mathrm{V}(\mathcal{Q})$. As usual, the coordinate ring $\mathbb{K}(\mathrm{V}(\mathcal{Q}))$ is

$$
\mathbb{K}(\mathrm{V}(\mathcal{d}))=\{[f] \mid f \in \mathbb{A}\} .
$$

Definition 2. (Localization of $\mathrm{V}(\mathcal{l})$ at a point) Let $\mathfrak{m}_{p}$ be the maximal ideal $\mathfrak{m}_{p}=$ $\left\langle x_{1}-p_{1}, \ldots, x_{n}-p_{k}\right\rangle \subset \mathbb{A}$ where $p=\left(p_{1}, \ldots, p_{n}\right) \in \mathrm{V}(\mathcal{l}) \subset \mathbb{K}^{n}$. Then we can write the localization of $\mathbb{A}$ at $p$ as

$$
\mathcal{O}_{p}=\left\{f / g \mid f \in \mathbb{A}, g \notin \mathfrak{m}_{p}\right\}
$$

The unique maximal ideal in this case is

$$
m_{p}=\left\{f / g \mid f \in \mathfrak{m}_{p}, g \notin \mathfrak{m}_{p}\right\}
$$

The localization of $\mathrm{V}(\ell)$ at $p$ is the ideal

$$
\mathcal{O}_{V, p}=\left\{f / g \mid f \in \mathbb{K}(\mathrm{V}(\mathcal{l})), g \notin \mathfrak{m}_{p}\right\} \subset \mathcal{O}_{p},
$$

Notice that $\mathcal{C} \subset \mathfrak{m}_{p}$ and $\mathcal{O}_{V, p}$ is a local ring which is a subring of a $\mathcal{O}_{p}$ with maximal ideal $\mathfrak{m}_{p} \mathcal{O}_{V, p}=M_{p}$. 


\section{SiNGULARITIES AND DIMENSION}

In this section we will briefly present the relevant definitions and theorems in order to compute our examples. Remember that the embedding dimension edim $\left(\mathcal{O}_{V, p}\right)=$ $\operatorname{dim}_{K}\left(M_{p} / M_{P}^{2}\right)$ of an algebraic variety is the minimal number of generators of $M_{p}$. Particulary important is that the Krull dimension of an ideal can be easily computed if the elements of the Gröbner basis of an ideal is known.

Definition 3 (Singular and regular points of a variety). Suppose that $d$ is a radical ideal. The local ring $\mathcal{O}_{V, p}$ is a regular local ring if

$$
\operatorname{dim}_{K}\left(\mathcal{O}_{V, p}\right)=\operatorname{edim}\left(\mathcal{O}_{V, p}\right)=\operatorname{dim}\left(T_{p} \mathrm{~V}(\mathcal{\ell})\right),
$$

where $T_{p} \mathrm{~V}(\ell)$ denotes the tangent space of $\mathrm{V}(\mathcal{Q})$ at $p$. If the point $p$ is not regular, it is singular.

The last equation in Definition 3 gives us the actual means to compute the singular points $[5,7,8,11]$.

Theorem 1 (Jacobian criterion). Let $d=\left\langle g_{1}, \ldots g_{n}\right\rangle \subset \mathbb{A}$ be a radical ideal and suppose that $\mathrm{V}(\mathcal{Q}) \subset \mathbb{K}^{k}$ is equidimensional ${ }^{*}$ and $\operatorname{dim}(\mathrm{V}(\mathcal{l}))=k-\ell$. Then the singular variety of $\mathrm{V}(\mathcal{Q})$ is

$$
S(\mathrm{~V}(\mathscr{d}))=\mathrm{V}\left(\mathscr{l}+\mathrm{I}_{l}(d g)\right)=\mathrm{V}(\mathscr{l}) \cap \mathrm{V}\left(\mathrm{I}_{l}(d g)\right) \subset \mathbb{K}^{k} .
$$

Here $\mathrm{I}_{l}(d g)$ denotes the $l$ th Fitting ideal of the Jacobian of the constraint map $g$ generated by $l \times l$ minors of $d g$. From this, it follows that, if $p \in S(\mathrm{~V}(\mathcal{d}))$, then $\mathcal{O}_{V, p}$ is not a regular local ring. Moreover, if $1 \in \mathcal{l}+\mathrm{I}_{l}(d g)$, then the variety $\mathrm{V}(\mathcal{l})$ is naturally smooth since $\mathrm{V}(1)=\varnothing$.

Let us then introduce another important object in our analysis - the tangent cone $[3$, 4]. We can rearrange any polynomial $f \in \mathbb{A}$ by total degree $d$ as a linear combination $f=f_{p, 0}+f_{p, 1}+\ldots+f_{p, d}$, where

$$
f_{p, k}=\sum_{|\alpha|=k} c_{\alpha}(x-p)^{\alpha} .
$$

Here, $\alpha$ is a multi index. Let us denote by $f_{p \text {,min }}$ the nonzero homogeneous component of smallest degree.

Definition 4 (tangent cone). Suppose that $\mathrm{V}(\mathcal{Q}) \subset \mathbb{K}^{k}$ is an affine variety and let $p \in \mathrm{V}(\ell)$. Let $d_{p \text {,min }}$ be the ideal generated by the minimal parts of polynomials in $\ell$. The tangent cone of $\mathrm{V}(\ell)$ at $p$, denoted by $C_{p}(\mathrm{~V}(\ell))$, is

$$
C_{p}(\mathrm{~V}(d))=\mathrm{V}\left(d_{p, \min }\right),
$$

The following theorem allows us also to distinguish between singular and regular points $[3,4]$.

${ }^{*}$ A variety is called equidimensional if all of its irreducible components have the same dimension. 
Theorem 2. The following conditions are equivalent:

(1) $p \in \mathrm{V}$ is a regular point of $\mathrm{V}$;

(2) $\operatorname{dim}\left(C_{p}(\mathrm{~V})\right)=\operatorname{dim}\left(T_{p} \mathrm{~V}\right)$;

(3) $C_{p}(\mathrm{~V})=T_{p} \mathrm{~V}$.

Finally, let us present a theorem to distinguish certain types of singularities [12].

Theorem 3. Suppose that $d \subset \mathbb{K}\left[x_{1}, \ldots, x_{k}\right]$ is a ideal where $\mathbb{K}$ is algebraically closed. Let $p \in \mathrm{V}(\mathcal{Q})$ be a singular point of $\mathrm{V}(\mathcal{Q})$ and $\mathcal{O}_{p}$ be the local ring at $p$. If the prime decomposition of the radical of $\mathcal{O}_{V, p}$ in the local ring is

$$
\sqrt{\mathcal{O}_{V, p}}=d_{1} \cap \ldots \cap d_{r} \subset \mathcal{O}_{p},
$$

then the corresponding irreducible varieties $\mathrm{V}\left(d_{i}\right)$ of prime ideals $d_{i}$ represent varieties passing through the singular point $p$ and the varieties intersect at this point. However, if the prime decomposition of $\mathcal{O}_{V, p}$ is $\sqrt{\mathcal{O}_{V, p}}=\mathcal{d}$, then $d$ is an integral domain and the point $p$ is a singularity of an irreducible variety $\mathrm{V}(\mathcal{d})$.

\section{EXAMPLES}

Here we apply the previous theorems to three easy examples.

\subsection{Simple slider-crank mechanisms}

Let us consider a mechanism constructed from two and three bars attached to each other with a revolute joint and suppose that the last bar is constrained to move on $x$-axis.
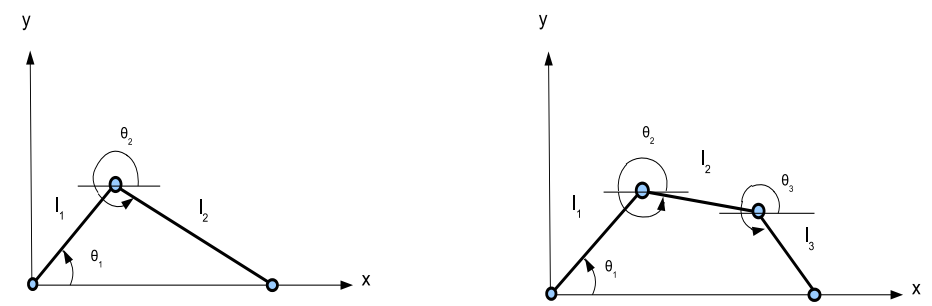

FigURE 1. On the left the 2-bar slider-crank mechanism. On the right the 3-bar slider crank mechanism. 


\subsubsection{2-bar slider-crank mechanism}

The constraint mapping $g: \mathbb{R}^{2} \mapsto \mathbb{R}$ for the 2-bar slider-crank mechanism is then

$$
g\left(\theta_{1}, \theta_{2}\right)=l_{1} \sin \left(\theta_{1}\right)+l \sin \left(\theta_{2}\right) .
$$

The configuration space is then the analytic variety $g^{-1}(0)$. With substitutions $c_{i}=$ $\cos \left(\theta_{i}\right)$ and $s_{i}=\sin \left(\theta_{i}\right)$, the constraint equations take the form

$$
p_{1}=l_{1} s_{1}+l_{2} s_{2}=0, p_{2}=c_{1}^{2}+s_{1}^{2}-1=0, p_{3}=c_{2}^{2}+s_{2}^{2}-1=0 .
$$

The configuration space is then $\mathrm{V}\left(\left\langle p_{1}, p_{2}, p_{3}\right\rangle\right)=\mathrm{V}(\mathcal{\ell})$. Now it is easy to check that $\mathscr{d}$ is a radical ideal and, moreover, $\operatorname{dim}(\mathrm{V}(\mathscr{d}))=1$. Let us then deduce the necessary condition for singularities from Theorem 1 in $\left(l_{1}, l_{2}\right)$-space. Since $\operatorname{dim}(\mathrm{V}(\mathscr{d}))=1$, the singular variety of $\mathrm{V}(\mathcal{l})$ is

$$
S(\mathrm{~V}(\mathscr{\ell}))=\mathrm{V}\left(\mathcal{l}+\mathrm{I}_{3}(d p)\right)
$$

Decomposing the Fitting ideal $I_{3}(d p)$ gives

$$
\sqrt{I_{3}(d p)}=d_{1} \cap \ldots \cap d_{6},
$$

Only the ideal $d_{5}=\left\langle c_{1}, c_{2}\right\rangle$ corresponds to a physically feasible solution so we only need to look at the intersection of $\mathrm{V}\left(\mathcal{l}_{5}\right)$ and $\mathrm{V}(\mathcal{l})$. To find the necessary condition, we compute the Gröbner basis of $S=d+d_{5}$ in the ring $\mathbb{Q}\left[\left(c_{1}, s_{1}, c_{2}, s_{2}\right),\left(l_{1}, l_{2}\right)\right]$ with the above elimination ordering. The computation shows

$$
E=S \cap \mathbb{Q}\left[l_{1}, l_{2}\right]=\left\langle\left(l_{1}+l_{2}\right)\left(l_{1}-l_{2}\right)\right\rangle,
$$

Hence $l_{1}=l_{2}$ is a necessary condition for singularities in $\left(l_{1}, l_{2}\right)$-space. Next we choose for example $l_{1}=l_{2}=1$ and compute the actual singular points. This gives

$$
\sqrt{S}=\left\langle s_{2}+1, c_{2}, s_{1}+s_{2}, c_{1}\right\rangle \cap\left\langle s_{2}-1, c_{2}, s_{1}+s_{2}, c_{1}\right\rangle .
$$

Thus the singular points are

$$
\mathrm{V}(S)=\mathrm{V}(\sqrt{S})=\{(0,1,0,-1),(0,-1,0,1)\}=q_{1} \cup q_{2} .
$$

Let us then carry out a local analysis for variety $\mathrm{V}(\mathscr{l})$ at the singular point $q_{2}=$ $\left(c_{1}, s_{1}, c_{2}, s_{2}\right)=(0,-1,0,1)$. Now we can compute the the tangent cone and get

$$
C_{q_{2}}(\mathrm{~V}(\mathcal{l}))=\mathrm{V}\left(\left\langle c_{1}, c_{2},\left(s_{1}-1\right)^{2}-\left(s_{2}+1\right)^{2}\right\rangle\right) .
$$

Near $q_{2}$, the variety $\mathrm{V}(\mathcal{Q})$ looks like two lines $s_{1}-1= \pm\left(s_{2}+1\right)$ intersecting in the plane $c_{1}=c_{2}=0$. Next, we consider $\mathscr{l}=\left\langle p_{1}, p_{2}, p_{3}\right\rangle$ in the local ring $\mathcal{O}_{q_{2}}$ using local ordering and compute the prime decomposition of $\mathcal{O}_{V, q_{2}}$. As expected, we have

$$
\mathcal{O}_{V, q_{2}}=H_{1} \cap H_{2} .
$$


By theorem 3, at least two irreducible varieties/motion modes pass through $q_{2}$. In fact, when we compute in global ordering after substitution $l_{1}=l_{2}=1$, we simply have

$$
\begin{aligned}
& \mathcal{l}=\mathcal{l}_{1} \cap \mathcal{l}_{2}=\left\langle c_{2}^{2}+s_{2}^{2}-1, c_{1}-c_{2}, s_{1}+s_{2}\right\rangle \cap\left\langle c_{2}^{2}+s_{2}^{2}-1, c_{1}+c_{2}, s_{1}+s_{2}\right\rangle, \\
& \mathrm{V}(\mathcal{l})=\mathrm{V}\left(\mathcal{l}_{1}\right) \cup \mathrm{V}\left(\mathcal{l}_{2}\right) \text { and } S(\mathrm{~V}(\mathcal{l}))=\mathrm{V}\left(\mathcal{l}_{1}\right) \cap \mathrm{V}\left(\mathcal{l}_{2}\right) .
\end{aligned}
$$

\subsubsection{3-bar slider-crank mechanism}

Let us then do similar analysis for 3-bar slider crank mechanism. With substitutions $c_{i}=\cos \left(\theta_{i}\right)$ and $s_{i}=\sin \left(\theta_{i}\right)$, as before, the constraint equations take the form

$$
p_{1}=l_{1} s_{1}+l_{2} s_{2}+l_{3} s_{3}=0, \quad p_{i+1}=c_{i}^{2}+s_{i}^{2}-1=0, \quad 1 \leq i \leq 3 .
$$

Again, we check that $\ell$ is radical and compute $\operatorname{dim}(\mathrm{V}(\ell))=2$. The singular variety is then

$$
S(\mathrm{~V}(\mathscr{l}))=\mathrm{V}\left(\mathcal{l}+\mathrm{I}_{4}(d p)\right) .
$$

Again, we first analyze the Fitting ideal $\mathrm{I}_{4}(d p)$ and find

$$
\sqrt{I_{4}(d p)}=d_{1} \cap \ldots \cap d_{11} .
$$

Only one of the prime components $d_{9}=\left\langle c_{1}, c_{2}, c_{3}\right\rangle$ is physically relevant so we compute the Gröbner basis of $S=d+d_{9}$ in the ring

$$
\mathbb{Q}\left[\left(c_{1}, s_{1}, c_{2}, s_{2}, c_{3}, s_{3}\right),\left(l_{1}, l_{2}, l_{3}\right)\right]
$$

with the above elimination ordering. Now we find

$$
\begin{aligned}
E & =S \cap \mathbb{Q}\left[l_{1}, l_{2}, l_{3}\right]=\left\langle\left(l_{1}+l_{2}+l_{3}\right)\left(l_{1}-l_{2}-l_{3}\right)\left(l_{1}+l_{2}-l_{3}\right)\left(l_{1}-l_{2}+l_{3}\right)\right\rangle \\
& =\left\langle h_{1} h_{2} h_{3} h_{4}\right\rangle
\end{aligned}
$$

The necessary conditions for singularities are thus $h_{2}=0, h_{3}=0$ or $h_{4}=0$. Let us choose $l_{1}=2$ and $l_{2}=l_{3}=1$ so that $h_{2}=0$ is fulfilled. This gives

$$
\sqrt{S}=\left\langle c_{1}, s_{1}-1, c_{2}, s_{2}+1, c_{3}, s_{3}+1\right\rangle \cap\left\langle c_{1}, s_{1}-1, c_{2}, s_{2}+1, c_{3}, s_{3}+1\right\rangle .
$$

The singular points are thus

$$
\mathrm{V}(S)=\mathrm{V}(\sqrt{S})=\{(0,1,0,-1,0,-1),(0,-1,0,1,0,1)\}=q_{1} \cup q_{2} .
$$

Let us then investigate locally the variety at point $q_{2}$. Computing the tangent cone gives

$$
C_{q_{2}}(\mathrm{~V}(\mathcal{d}))=\mathrm{V}\left(\left\langle c_{1}, c_{2}, c_{3}, 2\left(s_{1}+1\right)^{2}-\left(s_{2}-1\right)^{2}-\left(s_{3}-1\right)^{2}\right\rangle\right) .
$$


Note that $2\left(s_{1}+1\right)^{2}-\left(s_{2}-1\right)^{2}-\left(s_{3}-1\right)^{2}=0$ actually gives a cone in the $\left(s_{1}, s_{2}, s_{3}\right)$ space. Moreover, when we compute the prime decomposition of the radical of localization $\mathcal{O}_{V, q_{2}}$ of $\mathrm{V}(\mathcal{\ell})$ at $\mathcal{O}_{q_{2}}$, we find that

$$
\sqrt{\mathcal{O}_{V, q_{2}}}=\mathcal{O}_{V, q_{2}}
$$

Thus the singularity is not an intersection of different motion modes/irreducible varieties. It is still possible to visualize the configuration spaces of 2-bar and 3-bar slider crank mechanisms. In the first case, the configuration space "breaks" naturally to two irreducible varieties. In the 3-bar case, such separation does not exist.
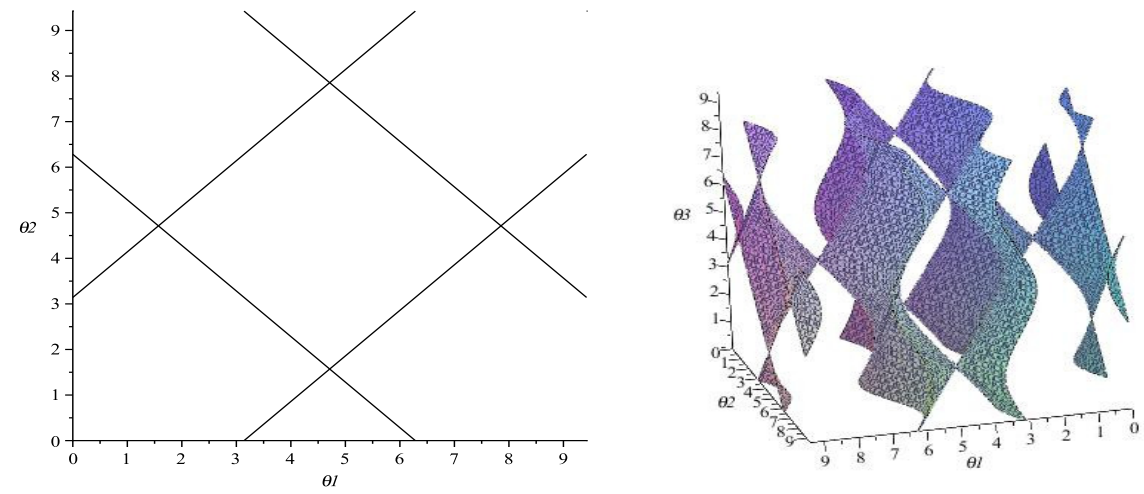

FIGURE 2. On the left, the configuration space of 2-bar slider-crank mechanism in the $\left(\theta_{1}, \theta_{2}\right)$-space. On the right, the configuration space of 3-bar slider crank mechanism in the $\left(\theta_{1}, \theta_{2}, \theta_{3}\right)$-space.

Remark 2. The plots agree with our computational results. Also, in the 2-bar slider crank case, there are no regular solutions $c: I \mapsto g^{-1}(0)$ for equations of motion (1.1) through the singular point $q_{2}=c\left(t_{0}\right)$ from one motion mode to another since, for such solutions, automatically, $c^{\prime}\left(t_{0}\right)=(0,0)$. However, in the case of the 3-bar slider crank, such a problem does not exist.

\subsection{Closed four bar mechanism}

Let us finally consider the closed four bar mechanism. To simplify the analysis, we assume that one bar with a length of unity is fixed on the $x$-axis.

In this case, the constraint equations are given by

$$
\begin{aligned}
p_{1}=l_{1} c_{1}+l_{2} c_{2}+l_{3} c_{3}-1 & =0, \\
p_{2}=l_{1} s_{1}+l_{2} s_{2}+l_{3} s_{3} & =0, \\
p_{2+i}=c_{i}^{2}+s_{i}^{2}-1 & =0, \quad 1 \leq i \leq 3 .
\end{aligned}
$$




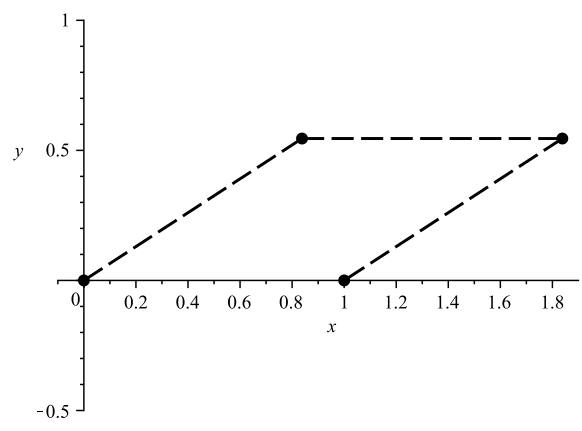

FIGURE 3. Simple closed four bar mechanism.

Setting $\mathscr{d}=\left\langle p_{1}, \ldots, p_{5}\right\rangle$, we compute $\operatorname{dim}(\mathrm{V}(\mathcal{l}))=1$. The singular variety is thus

$$
S(\mathrm{~V}(\mathscr{\ell}))=\mathrm{V}\left(\mathcal{l}+\mathrm{I}_{5}(d p)\right) .
$$

The prime decomposition of $\sqrt{I_{5}(d p)}$ yields

$$
\sqrt{I_{5}(d p)}=d_{1} \cap \ldots \cap d_{10}
$$

The only physically relevant component is given by

$$
d_{8}=\left\langle-s_{2} c_{3}+c_{2} s_{3},-s_{1} c_{3}+c_{1} s_{3},-s_{1} c_{2}+c_{1} s_{2}\right\rangle
$$

Computing the Gröbner basis of $S=d+d_{8}$ in the ring

$$
\mathbb{Q}\left[\left(c_{1}, s_{1}, c_{2}, s_{2}, c_{3}, s_{3}\right),\left(l_{1}, l_{2}, l_{3}\right)\right]
$$

we find

$$
E=S \cap \mathbb{Q}\left[l_{1}, l_{2}, l_{3}\right]=\left\langle k_{1} \cdot \ldots \cdot k_{9}\right\rangle .
$$

The necessary condition for $\mathrm{V}(S) \neq \varnothing$ in $\left(l_{1}, l_{2}, l_{3}\right)$-space is $k_{i}=0$, where the nine polynomials $k_{i}$ are of the form

$$
k_{i}=l_{1} \pm l_{2} \pm l_{3} \pm 1
$$


Let us choose $l_{1}=l_{2}=l_{3}=1$ so that $k_{4}=l_{1}-l_{2}+l_{3}-1=0$. After substitutions we compute

$$
\begin{aligned}
\sqrt{S} & =\mathscr{g}_{1} \cap \mathscr{g}_{2} \cap \mathscr{g}_{3} \\
\mathcal{J}_{1} & =\left\langle c_{1}+1, s_{1}, c_{2}-1, s_{2}, c_{3}-1, s_{3}\right\rangle \\
\mathcal{g}_{2} & =\left\langle c_{1}+1, s_{1}, c_{2}-1, s_{2}, c_{3}+1, s_{3}\right\rangle \\
\mathcal{J}_{3} & =\left\langle c_{1}+1, s_{1}, c_{2}+1, s_{2}, c_{3}-1, s_{3}\right\rangle .
\end{aligned}
$$

Also, after substitutions, the constraint ideal decomposes as $\sqrt{d}=d^{\prime}=d_{1} \cap d_{2} \cap d_{3}$ and easy computation shows that the singularities $q_{i}$ are the intersections

$$
\begin{aligned}
& q_{1}=\mathrm{V}\left(\mathscr{g}_{1}\right)=\mathrm{V}\left(d_{1}\right) \cap \mathrm{V}\left(d_{3}\right)=(-1,0,1,0,1,0) \\
& q_{2}=\mathrm{V}\left(\mathscr{g}_{2}\right)=\mathrm{V}\left(d_{2}\right) \cap \mathrm{V}\left(d_{3}\right)=(1,0,1,0,-1,0) \\
& q_{3}=\mathrm{V}\left(\mathcal{g}_{3}\right)=\mathrm{V}\left(d_{1}\right) \cap \mathrm{V}\left(d_{2}\right)=(1,0,-1,0,1,0) .
\end{aligned}
$$

Let us still do the local analysis for $\mathrm{V}(\mathcal{Q})$ for example at $q_{1}$. The tangent cone $C_{q_{1}}(\mathrm{~V}(\mathcal{Q}))$ is

$$
C_{q_{1}}(\mathrm{~V}(\mathcal{l}))=\mathrm{V}\left(\left\langle s_{1}, s_{2}, s_{3}, c_{1}+c_{2}+c_{3}-1,\left(c_{2}-1\right)\left(c_{3}-1\right)\right\rangle\right)
$$

The singularity looks now again like the intersection of two lines in the hyper plane $s_{1}=s_{2}=s_{3}=0$. Then, the computation in the local ring shows that

$$
\mathcal{O}_{V, q_{1}}=H_{1} \cap H_{2}
$$

This confirms that the two irreducible varieties $\mathrm{V}\left(\mathscr{l}_{1}\right)$ and $\mathrm{V}\left(\mathcal{l}_{2}\right)$ intersect at point $q_{1}$ as Theorem 3 suggests.
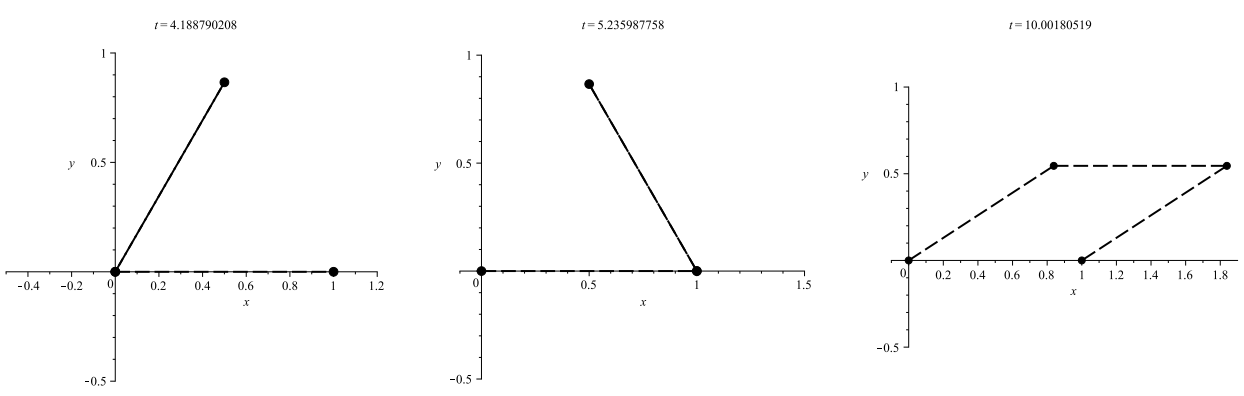

FIGURE 4 . On the left the degenerate motion mode V $\left(\mathscr{d}_{1}\right)$. In the middle the degenerate motion mode $\mathrm{V}\left(d_{2}\right)$. On the right the "actual" four bar motion mode $\mathrm{V}\left(\mathscr{d}_{3}\right)$. In both degenerate motion modes, two bars are fixed on the horizontal axis and two bars rotate freely together. In the "actual" motion mode, the mechanism rotates as one parallellogram. 
Remark 3. Notice again that there are no regular solutions $c: I \mapsto g^{-1}(0)$ for (1.1) which would take the the mechanism through $q_{1}$ from $\mathrm{V}\left(\ell_{3}\right)$ to $\mathrm{V}\left(\ell_{1}\right)$ and again through $q_{2}$ from $\mathrm{V}\left(\ell_{3}\right)$ to $\mathrm{V}\left(\ell_{1}\right)$. At singular points $c\left(t_{i}\right)=q_{i}, 1 \leq i \leq 3$, automatically, $c^{\prime}\left(t_{i}\right)=(0,0,0)$ since the one dimensional varieties intersect transversally.

\section{CONCLUSiOnS}

In this paper, we successfully analyzed configuration spaces of three simple mechanisms with computational algebraic geometry. The methods can be applied to more complicated mechanisms as well $[1,2,15]$. When we are able to represent the configuration space as an algebraic variety, we can use the tools of computational algebraic geometry to make both global and local statements about the configuration space. The local analysis for analytic varieties has been previously investigated in $[13,14]$ and the Gröbner bases methods in [6]. The dimension or mobility in particular motion mode $\mathrm{V}(\ell)$ can be regarded as the Krull dimension of the corresponding ideal $\ell$. The Jacobian criterion together with elimination theory provides us with the means to determine whether and under which conditions, configuration space singularities exist. The tangent cone and the localization of motion mode $\mathrm{V}(\mathcal{l})$ at a singular point gives us the means to investigate the nature of the singularity.

\section{REFERENCES}

[1] T. Arponen, S. Piipponen, and J. Tuomela, "Analysis of singularities of a benchmark problem," Multibody System Dynamics, vol. 19, no. 3, pp. 227-253, 2008.

[2] T. Arponen, S. Piipponen, and J. Tuomela, "Kinematic analysis of Bricard's mechanism," Nonlinear Dynamics, vol. 56, no. 1-2, pp. 85-99, 2009.

[3] D. Cox, J. Little, and D. O'Shea, Using algebraic geometry, 2nd ed., ser. Graduate Texts in Mathematics. New York: Springer, 2005, vol. 185.

[4] D. Cox, J. Little, and D. O'Shea, Ideals, varieties, and algorithms, 3rd ed., ser. Undergraduate Texts in Mathematics. New York: Springer, 2007, An introduction to computational algebraic geometry and commutative algebra.

[5] W. Decker and C. Lossen, Computing in algebraic geometry, ser. Algorithms and Computation in Mathematics. Berlin: Springer-Verlag, 2006, vol. 16, A quick start using SINGULAR.

[6] A. K. Dhingra, A. N. Almadi, and D. Kohli, "Closed-form displacement and coupler curve analysis of planar multi-loop mechanisms using Gröbner bases," Mech. Mach. Theory, vol. 36, no. 2, pp. 273-298, 2001.

[7] D. Eisenbud, Commutative algebra, ser. Graduate Texts in Mathematics. New York: SpringerVerlag, 1995, vol. 150, With a view toward algebraic geometry.

[8] G.-M. Greuel and G. Pfister, A singular introduction to commutative algebra. Berlin: SpringerVerlag, 2002, With contributions by Olaf Bachmann, Christoph Lossen and Hans Schn̈emann, With 1 CD-ROM (Windows, Macintosh, and UNIX).

[9] G.-M. Greuel, G. Pfister, and H. Schönemann, "Singular 3.0, A Computer Algebra System for Polynomial Computations," Centre for Computer Algebra, University of Kaiserslautern, 2005. [Online]. Available: http://www.singular.uni-kl.de

[10] K. Hulek, Elementary algebraic geometry, ser. Student Mathematical Library. Providence, RI: American Mathematical Society, 2003, vol. 20. 
[11] M. Kreuzer and L. Robbiano, Computational commutative algebra 2. Berlin: Springer-Verlag, 2005.

[12] H. Matsumura, Commutative ring theory, 2nd ed., ser. Cambridge Studies in Advanced Mathematics. Cambridge: Cambridge University Press, 1989, vol. 8.

[13] A. Müller, "Generic mobility of rigid body mechanisms," Mech. Mach. Theory, vol. 44, pp. 1240$1255,2009$.

[14] A. Müller and J. M. Rico, "Mobility and higher order local analysis of the configuration space of single-loops mechanisms," Advances in Robot Kinematics, vol. 4, pp. 215-224, 2009.

[15] S. Piipponen, "Singularity analysis of planar linkages," Multibody System Dynamics, vol. 22, no. 3, pp. 223-243, 2009.

Authors' addresses

\section{Samuli Piipponen}

University of Eastern Finland, Department of Physics and Mathematics, Yliopistokatu 2, P.O. Box 111, Joensuu, Finland

E-mail address: samuli.piipponen@uef.fi

\section{Jukka Tuomela}

University of Eastern Finland, Department of Physics and Mathematics, Yliopistokatu 2, P.O. Box 111, Joensuu, Finland

E-mail address: jukka.tuomela@uef.fi 